\title{
Lateral Coupling within the Ensemble of InAs/InGaAlAs/InP Quantum Dashes
}

\author{
K. RYCZKO, G. SĘK AND J. MisiewicZ \\ Institute of Physics, Wrocław University of Technology, Wybrzeże Wyspiańskiego 27, 50-370 Wrocław, Poland

\begin{abstract}
In this work we investigate the electronic structure of coupled quantum dashes. The respective confined state energy levels are calculated for various cross-sectional shapes and sizes of the dashes and in function of the lateral distance between them. The results are confronted with the existing experimental data on the optical transitions separation) the obtained direct coupling is weak and in most of the applications the dashes can be considered individually, as long as the ensemble is strongly inhomegeneous.
\end{abstract} \\ in such structures. It has been concluded that for realistic system parameters (geometry and spatial in-plane
}

DOI: 10.12693/APhysPolA.124.805

PACS: 78.55.Cr, 73.21.Hb, 78.67.Uh

\section{Introduction}

Semiconductor quantum dashes (QDashes) can be fabricated in the InAs-InP material system by molecular beam epitaxy. They have been proven to be advantageous when employed as the active region of near infrared photonic devices, especially when considering telecommunication and data communication applications. They have been demonstrated to be application-relevant in lasers and optical amplifiers, as offering high gain, tunable or polarization insensitive emission, especially at fiber-based optoelectronics wavelengths [1].

Furthermore, preliminary work on single quantum dashes [2-4] and their potential for emitters in quantum-electrodynamics-based experiments has recently been performed, revealing a possibility of realizing single photon sources at the telecommunication wavelengths. In all the cases, the electronic structure is discussed in a picture of independent dashes, in spite of typically very high surface density of these structures (even above $10^{11} \mathrm{~cm}^{-2}$ ), making the average in-plane distances significantly smaller than the dash characteristic sizes. This makes possible the existence of finite or even efficient carrier tunneling between the dashes, which could significantly affect both the ensemble properties in the laser active region, as this opens a lateral carrier transport channel, as well as the subtle properties of single dashes, due to effective weakening of the confinement, modification of the carrier wave functions and hence the exciton oscillator strength.

Therefore, investigation of the electronic structure of coupled quantum dashes is a basis for further understanding of the phenomena that occur in these structures and can have a substantial influence on their application potential. In this work, we discuss the electronic structure of the system composed of coupled InAs quantum-wire-like structures (the confinement in the longest dimension is neglected for simplicity), separated laterally by a thin InGaAlAs barrier lattice matched to the InP substrate. The respective confined state energy levels and the carrier probability densities are calculated within the $k \cdot p$ theory for different cross-sectional shapes and sizes of the dashes, and in function of the lateral distance between them. The results are confronted with the existing experimental data on the optical transitions in such structures $[5,6]$.

\section{Theoretical framework}

The self-organized InAs quantum dashes grown on $\mathrm{In}_{0.53} \mathrm{Ga}_{0.23} \mathrm{Al}_{0.24} \mathrm{As} / \mathrm{InP}$ are typically obtained by the molecular beam epitaxy technique. Strongly elongated structures in [1-10] direction are formed due to a relatively low strain (lattice mismatch of $3.4 \%$ ) and an anisotropic surface diffusion of atoms [7]. We model them here as being ideal triangles in the cross-section (see Fig. 1) and placed in a close lateral vicinity, treating both the QDash size/shape and the separation distance as main tuning parameters in the calculations.

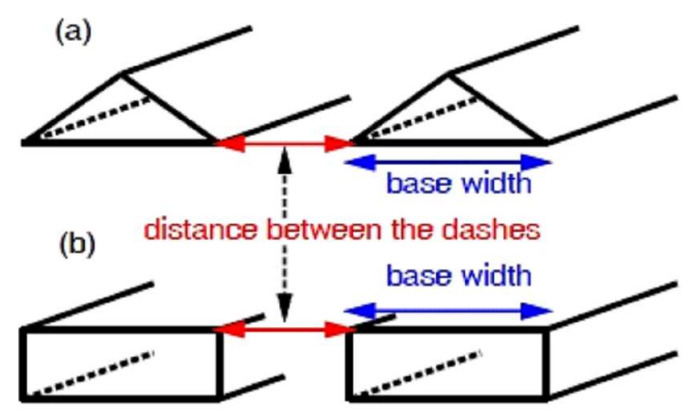

Fig. 1. Theoretical model of the laterally coupled (a) triangular and (b) rectangular quantum dashes with the specific height, base width, and distance between the dashes.

To use realistic system parameters, the length of quantum dashes can roughly be estimated to values between close to $100 \mathrm{~nm}$ to even several hundred $\mathrm{nm}$ [1]. The quantum dashes shape in the cross-section can be well 
approximated by a triangle. Their height and the base width determined by STEM in Ref. [7] are about 2-6 nm and $12-33 \mathrm{~nm}$, respectively. To investigate the shape effect in part at least, we considered and compared two geometries, triangular and rectangular quantum dashes at a certain distance from each other, as illustrated in Fig. 1.

To calculate the electronic structure for electrons and holes in coupled quantum dashes we solve two-dimensional Schrödinger equation, neglecting the confinement in the elongation direction [8]. In our analysis we assume finite barriers and different effective masses for the dash and barrier materials. The effect of strain is also included [9]. The energies and wave functions of the confined states are obtained numerically by using the finite difference method [10].

All material parameters were taken from Ref. [11] for $10 \mathrm{~K}$. The conduction band offset between $\mathrm{In}_{0.53} \mathrm{Ga}_{0.23} \mathrm{Al}_{0.24} \mathrm{As}$ and InAs is assumed to be $70 \%$ [12].

\section{Results and discussion}

We calculate first the transition energies of a single quantum dash of a triangular cross-section to model the real and existing object, and to simulate the available experimental data. In Fig. 2 we compare the calculated transition energies in a function of the base width with the experimental data from Ref. [6], extracted from photoreflectance spectra on the ensemble of various sized dashes.

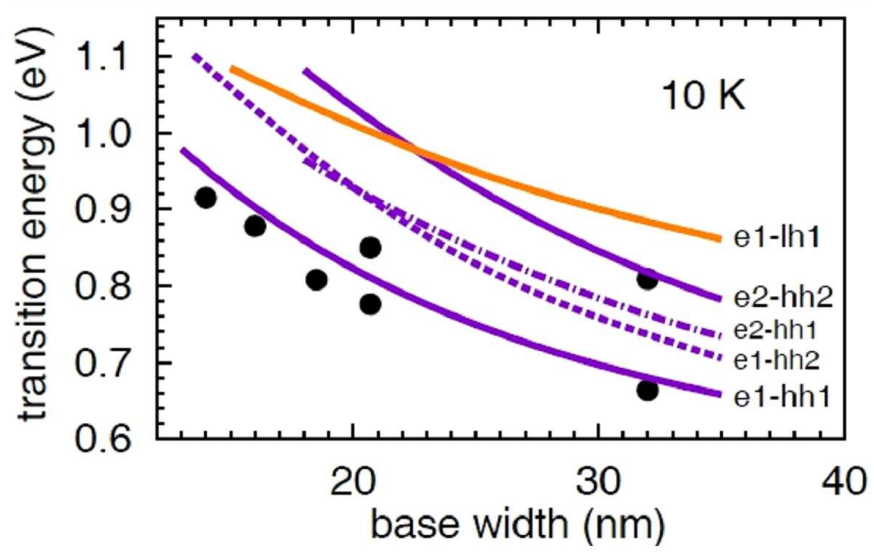

Fig. 2. The transition energies calculated (solid, dash and dash-dot lines) and measured experimentally (symbols) as a function of the base width in $\mathrm{In}_{0.53} \mathrm{Ga}_{0.23} \mathrm{Al}_{0.24} \mathrm{As} / \mathrm{InP}$ triangular quantum dashes. The experimental data are taken from Refs. [5, 6].

For all the experimentally investigated samples the lowest energy transition has been attributed to the transition between fundamental electron and heavy hole states, and it shifts from about $0.95 \mathrm{eV}$ down to $0.65 \mathrm{eV}$, when the base width of the dashes increases from $13 \mathrm{~nm}$ to $33 \mathrm{~nm}$. For the larger of those structures, there appears also an additional higher spectral feature which can in general be related to excited state transitions in the dashes.

The main goal of this comparison was the verification of the model as an approximation itself and the used material parameters. The agreement is not very exact due to neglecting the possible coupling and other details (as e.g. band mixing effects, Coulomb interaction, etc.), but in the first approximation it allows using the model in the further considerations, to simulate the strength of the in-plane coupling.

Regarding the latter, we started with calculations of the coupling for two triangular and rectangular dashes, when a distance from each other is fixed and for the increasing QDash base width. In Fig. 3, there is shown the dependence of the splitting energy between the ground and first excited states for electrons as a function of the base width of the quantum dash.

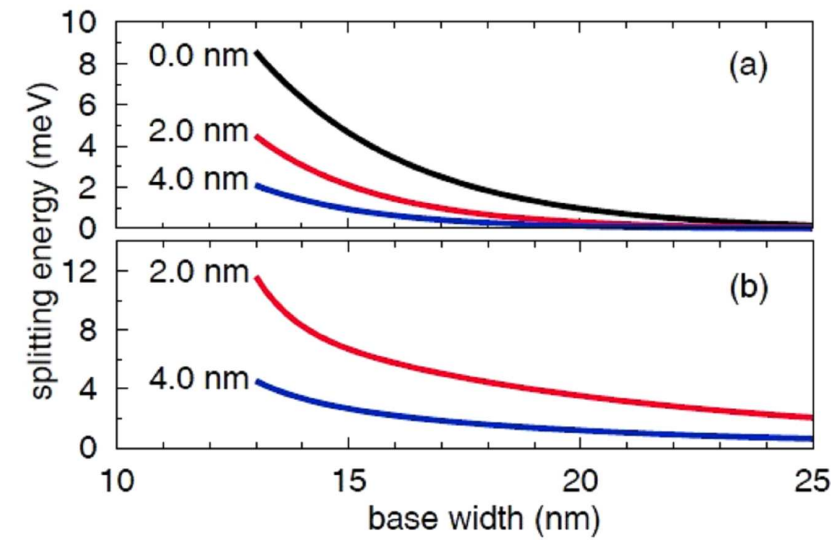

Fig. 3. The splitting energy between the ground and first excited state for electrons as a function of the base width in $\mathrm{In}_{0.53} \mathrm{Ga}_{0.23} \mathrm{Al}_{0.24} \mathrm{As} / \mathrm{InP}$ coupled (a) triangular, (b) rectangular quantum dashes. The black, red, and blue solid lines correspond to coupled quantum dashes with the lateral separating distance equal to 0 , 2 , and $4 \mathrm{~nm}$, respectively.

This has been studied for the base widths changed from $10 \mathrm{~nm}$ to $25 \mathrm{~nm}$, and for three different separation distances: 0,2 , and $4 \mathrm{~nm}$. The range of distances is chosen based on the structural data [7], i.e. being indeed usually not beyond that range.

Figure $3 \mathrm{a}$ and $\mathrm{b}$ shows the results for the used triangular and rectangular shapes in the cross-section. For both the cases the splitting energy, being a figure of merit of the strength of the quantum-mechanical coupling, decreases significantly with the base width getting down to single meV range for the widths above $20 \mathrm{~nm}$ already. This decreases faster for the triangular dashes, as they are effectively less coupled. For the smallest widths the splitting energy is in the range of $2-8 \mathrm{meV}$ and $4-14 \mathrm{meV}$ for the triangular and rectangular shapes, respectively, indicating again a much weaker coupling for the former. This effect is due to a different behavior of the electron wave function (probability density distribution) of 
the first two electron states. In the triangular quantum dashes, the electron probability density is more localized in each dash, and hence leaking less into the neighboring dash, decreasing the overall carrier tunneling probability. In contrast, in the rectangular quantum dashes, the states of the neighboring dashes will feel each other more, significantly enhancing the coupling.

Figure 4 shows the calculated splitting energy versus the distance between the dashes. In our calculation the base width is equal to $14 \mathrm{~nm}$ for the triangular quantum dashes and $14 \mathrm{~nm}$ and $32 \mathrm{~nm}$ for the rectangular quantum dashes.

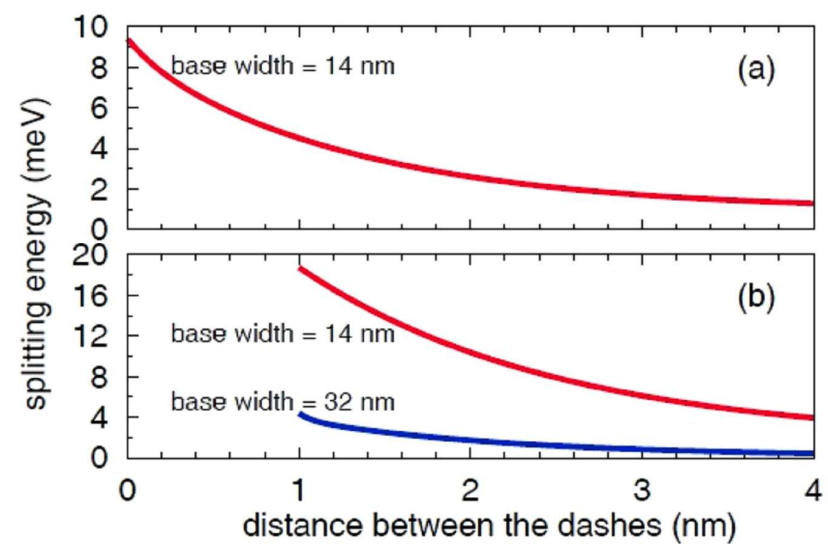

Fig. 4. The splitting energy between ground and the first excited states for electrons as a function of the distance between the dashes in $\operatorname{In}_{0.53} \mathrm{Ga}_{0.23} \mathrm{Al}_{0.24} \mathrm{As} / \mathrm{InP}$ coupled (a) triangular, (b) rectangular quantum dashes. The red and blue solid lines correspond to coupled quantum dashes with the base width 14 and $32 \mathrm{~nm}$, respectively.

When the distance between the QDashes becomes larger, the splitting energy decreases, as expected. Moreover, the values of the splitting energy for the rectangular quantum dashes is more than twice larger than for the triangular quantum dashes. From Fig. 4a we can see that in order to get a splitting energy value which would at least count in the single dash considerations and possible applications, they would need to touch each other in fact, giving a noticeable coupling (about $10 \mathrm{meV}$ ). The latter would still be hardly distinguishable in all the experiments and applications concerning the ensemble of dashes experiencing rather large inhomogeneous broadening of the optical transitions in all the spectral response functions being on the level of $50 \mathrm{meV}$. For the separation distance of about $3 \mathrm{~nm}$, which is quite typical for coupled InAs $/ \mathrm{In}_{0.53} \mathrm{Ga}_{0.23} \mathrm{Al}_{0.24} \mathrm{As} / \mathrm{InP}$ quantum dashes within the ensemble [7], the splitting energy is around $2 \mathrm{meV}$ only, in the case of the base width equal to $14 \mathrm{~nm}$. For larger base widths this becomes almost fully negligible, and would actually not be seen in the energy scale of Fig. 4.

In order to relate these energy level splitting values with the efficiency of the tunneling process between the dashes, they can be converted into the related tunneling rates or tunneling times according to the common handbook formulae [13]. We have obtained that in the case of two identical quantum dashes with triangular cross-section, the base width of $14 \mathrm{~nm}$ and the lateral separation equal to $0 \mathrm{~nm}$ (i.e. the maximal possible coupling) the tunneling time is about $1 \mathrm{ps,}$ which is very short compared to the typical lifetimes of the order of $1 \mathrm{~ns}$. In the real ensemble however, the dashes are never identical, or actually in these structures on InP substrate the distribution of sizes and compositions is rather large, as mentioned above. Therefore, after introducing the asymmetry in the sizes of the dashes the times increase significantly to above $200 \mathrm{ps}$ when one of the dashes has a twice larger base width, but still for the $0 \mathrm{~nm}$ distance between the dashes. Eventually, as the zero distance is a very ultimate case, this should be rather kept in the range of a few nm. For the separation between the dashes of about $3 \mathrm{~nm}$ (and two different dashes as above) the related tunneling time increases to above $2 \mathrm{~ns}$ already, i.e. transfer becomes inefficient compared to the radiative recombination. It is also worth noticing that similar calculations for the heavy hole states give even smaller splitting (by almost an order of magnitude) and much larger tunneling times, therefore can actually be ignored when discussing the coupling effects and their influence on the optical spectra. This is why the results for the holes have not been shown here nor discussed more in detail.

Taking all the abovementioned into account, the efficiency of the tunneling and the carrier transfer between the quantum dashes will mostly depend on the ensemble uniformity and for the typically strongly inhomogeneous system is expected to be low.

\section{Conclusions}

In this work, we investigated the electronic structure of coupled quantum dashes to simulate the conditions occurring usually in the practically realized systems with these strongly asymmetric structures in the InAs/InP material system. The single-band effective-mass calculations including strain showed that for lateral coupling within the ensemble of $\mathrm{InAs} / \mathrm{In}_{0.53} \mathrm{Ga}_{0.23} \mathrm{Al}_{0.24} \mathrm{As} /$ InP triangular quantum dashes the splitting energy between the ground and first excited states of electron should be expected to be smaller than $2 \mathrm{meV}$, which corresponds to picosecond transfers time between the neighbouring and very closely spaced quantum dashes. However, the tunneling rates will decrease by more than three orders of magnitude for more realistic case of two dashes significantly differing in size and separated by a few nanometers. Therefore, it can eventually be concluded that for the system parameters (geometry and spatial in-plane separation) corresponding to the existing InAs/InP QDash structures obtained direct coupling is indeed weak and in most of the applications the dashes can be considered individually, as long as the ensemble is strongly inhomogeneous. 


\section{Acknowledgments}

This work was supported by a project of the $\mathrm{Na}$ tional Science Centre of Poland within grant No. 2011/ 02/A/ST3/00152.

\section{References}

[1] J.P. Reithmaier, G. Eisenstein, A. Forchel, Proc. IEEE 95, 1779 (2007).

[2] G. Sęk, P. Podemski, A. Musiał, J. Misiewicz, S. Hein, S. Höfling, A. Forchel, J. Appl. Phys. 105, 086104 (2009).

[3] T. Mensing, L. Worschech, R. Schwertberger, J.P. Reithmaier, A. Forchel, Appl. Phys. Lett. 82, 2799 (2003).

[4] N. Chauvin, P. Nedel, C. Seassal, B. Ben Bakir, X. Letartre, M. Gendry, A. Fiore, P. Viktorovitch, Phys. Rev. B 80, 045315 (2009).

[5] W. Rudno-Rudzinski, R. Kudrawiec, P. Podemski, G. Sęk, J. Misiewicz, A. Somers, R. Schwertberger, J.P. Reithmaier, A. Forchel, Appl. Phys. Lett. 89, 031908 (2006).

[6] G. Sęk, P. Podemski, R. Kudrawiec, J. Misiewicz, A. Somers, S. Hein, S. Höfling, J.P. Reithmaier, A. Forchel, Proc. SPIE 6481, 64810F (2007).
[7] A. Sauerwald, T. Kümmell, G. Bacher, A. Somers, R. Schwertberger, J.P. Reithmaier, A. Forchel, Appl. Phys. Lett. 86, 253112 (2005).

[8] M. Gioannini, IEEE J. Quantum Electron. 40, 364 (2004).

[9] G.L. Bir, G.E. Pikus, Symmetry and Strain-Induced Effects in Semiconductors, Wiley, New York 1974.

[10] J.W. Thomas, Numerical Partial Differential Equations, Finite Difference Methods, Springer-Verlag, New York 1995.

[11] I. Vurgaftman, J.R. Meyer, L.R. Ram-Mohan, J. Appl. Phys. 89, 5815 (2001).

[12] W. Rudno-Rudziński, R. Kudrawiec, G. Sęk, J. Misiewicz, A. Somers, R. Schwertberger, J.P. Reithmaier, A. Forchel, Appl. Phys. Lett. 88, 141915 (2006).

[13] G. Bastard, Wave Mechanics Applied to Semiconductor Heterostructures, Les Éditions de Physique, Zone Industrielle de Courtabouf (France) 1988. 